\title{
Tratamiento tópico con Imiquimod crema al 5\% de la enfermedad de Bowen del pene
}

\author{
E. de Diego Rodríguez, A. Villanueva Peña, A. Hernández Castrillo, J.Mª Gómez Ortega* \\ Servicio de Urología. *Servicio de Anatomía-patológica. Hospital Comarcal de Laredo (Cantabria).
}

Actas Urol Esp 2005; 29 (8): 797-800

\section{RESUMEN}

TRATAMIENTO TOPICO CON IMIQUIMOD CREMA AL 5\% DE LA ENFERMEDAD DE BOWEN DEL PENE

Presentamos una alternativa más de tratamiento para las neoplasias intraepiteliales de pene (enfermedad de Bowen) mediante la aplicación tópica de imiquimod crema al 5\% 3 días alternos a la semana durante 5 semanas. El imiquimod es una imidazoquinolona, modificador de la respuesta inmune con efectos antiviral y antitumoral indirectos, que presenta escasos efectos secundarios a nivel local y que puede constituir una opción más en el tratamiento de la enfermedad de Bowen del pene.

Palabras clave: Carcinoma in situ. Neoplasia de pene. Neoplasia intraepitelial.

\section{ABSTRACT}

TREATMENT OF BOWEN'S DISEASE OF THE PENIS WITH IMIQUIMOD 5\% CREAM

We present an alternative treatment of the intraepithelial neoplasms of penis (Bowen's disease), the topical application of imiquimod $5 \%$ cream. It was applied 3 times weekly for 5 weeks. Imiquimod is an imidazoquinolone immune response modifier that has been shown to have indirect antiviral and antitumor effects with few adverse effects. Imiquimod 5\% cream may represent an alternative treatment option for Bowen`s disease.

Keywords: Carcinoma in situ. Penile neoplasms. Intraepithelial neoplasms.

$\mathrm{L}$ as neoplasias intraepiteliales a nivel del pene han sido descritas en la literatura indistintamente con varios nombres: eritroplasia de Queyrat, enfermedad de Bowen, carcinoma in situ, lo cual genera a menudo confusión.

La eritroplasia de Queyrat fue descrita inicialmente en 1891 por Tarnovsky y debe su nombre a Queyrat quien en 1911 aplica este término a unas lesiones rojizas, en forma de placa sobreelevada, bien delimitadas, localizadas en pene ${ }^{1}$. Sulzberger y Satenstein asocian histológicamente esta entidad a un carcinoma in situ de células escamosas $^{2}$.

En 1912 Bowen describe en 2 pacientes unas lesiones muy similares a la eritroplasia de Queyrat, localizadas en extremidades inferiores, que tam- bién podían aparecer en pene, constituyendo histológicamente un carcinoma in situ de células cutáneas $^{1,2}$.

Por lo tanto, si ambas entidades constituyen propiamente un carcinoma in situ desde el punto de vista histológico, el término de lesiones preneoplásicas, ampliamente utilizado, no sería correcto y sí quizá más apropiado el de neoplasia intraepitelial, siendo por tanto dos entidades potencialmente invasivas a nivel local que requieren tratamiento ${ }^{1,2}$.

La enfermedad de Bowen extragenital parece estar relacionada etiológicamente con la exposición crónica al sol y el contacto con arsénico y derivados; en el caso de la enfermedad genital es la infección por Papiloma virus el agente etiológi- 
co más implicado ${ }^{3-5}$ (fundamentalmente el serotipo 16 , en menor porcentaje el $18 \mathrm{y}$ a gran distancia otros serotipos $31,33,35,39,51$ ). Wieland ${ }^{6}$ argumenta que la presencia del serotipo 8 pudiese ayudar a diferenciar una eritroplasia de Queyrat de una enfermedad de Bowen.

Las opciones de tratamiento de estas lesiones son múltiples ${ }^{1,7,8}$ con resultados estéticos, funcionales y de curación variables y así se ha recurrido tradicionalmente a la exéresis de la lesión, criocirugía, radioterapia, aplicación tópica de 5-fluoruracilo y más recientemente laserterapia.

Presentamos una alternativa más de tratamiento de las neoplasias intraepiteliales de pene para el urólogo, consistente en la aplicación tópica de imiquimod crema al $5 \%$, un potente modulador de la respuesta inmune, no habiendo encontrado en la revisión de la literatura urológica ninguna referencia a esta opción terapéutica.

\section{CASO CLÍNICO}

Paciente varón de 68 años de edad que acude a consulta por disfunción eréctil de varios meses de evolución.

Como antecedentes de interés únicamente destacan hipertensión arterial, psoriasis e hiperuricemia en tratamiento médico.

A la exploración llama la atención una lesión enrojecida, brillante, sobreelevada y friable en cara dorsal de glande hasta surco balano-prepucial (Fig. 1), a la cual el paciente no daba importancia dado que no le originaba sintomatología alguna.

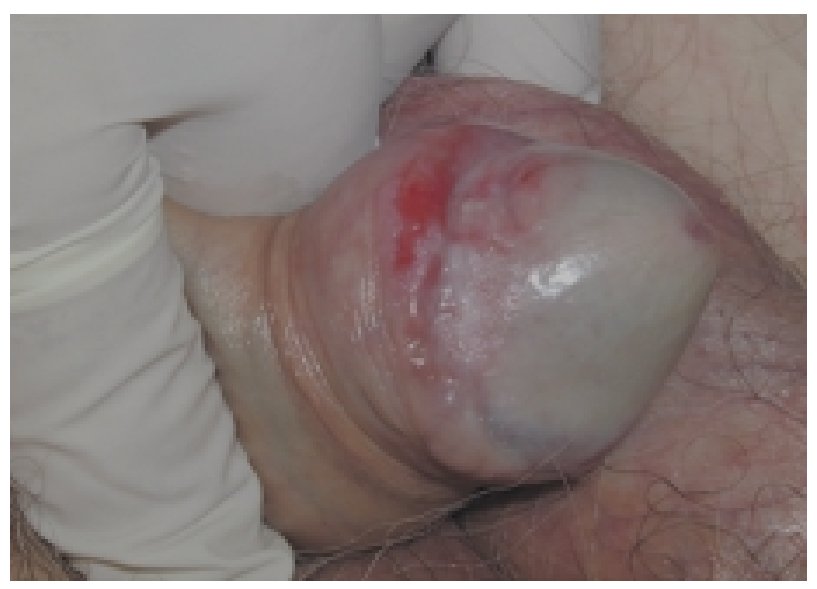

FIGURA 1. Aspecto macroscópico de lesión peneana.
Se practicó biopsia bajo anestesia local de dicha zona, cuyo informe anátomo-patológico fue de neoplasia intraepitelial de pene (enfermedad de Bowen), mostrándose un epitelio engrosado con células hipercromáticas y atípicas (Fig. 2).

Se instauró tratamiento tópico con imiquimod crema al 5\%, 3 aplicaciones semanales a días alternos durante 5 semanas.

La tolerancia al tratamiento fue buena, apareciendo fundamentalmente reacciones a nivel local del tipo prurito y sensación de quemazón, eritema y descamación durante las primeras aplicaciones y también adherencias prepuciales referidas por el paciente como muy molestas, por lo que se le practicó circuncisión al inicio del tratamiento.

A los 15 días de finalizadas las aplicaciones se revisó la lesión presentando una franca mejoría estética (Fig. 3), habiendo desaparecido los efectos secundarios del tratamiento.

Se realizó nueva biopsia de la zona tratada confirmándose la ausencia de atípias celulares en el epitelio, presentando una intensa reacción inflamatoria crónica (Fig. 4).

A los 6 meses de seguimiento del paciente no aparecieron signos clínicos de recidiva en lugar de la lesión.

\section{DISCUSIÓN}

La enfermedad de Bowen del pene constituye un carcinoma in situ desde el punto de vista histológico con mayor riesgo de evolución hacia un carcinoma invasivo (10\%) que el Bowen extragenital $^{7,8}$.

La biopsia de la lesión nos aportará el diagnóstico y la vez nos diferenciará esta lesión de la balanitis plasmocelular o balanitis de Zoom, entidad benigna, cuyo tratamiento se limita en general a la circuncisión. ${ }^{8}$

En 1999 Cox y Morton ${ }^{7}$ establecen las guías de tratamiento para la enfermedad de Bowen genital: escisión, criocirugía, cirugía de Mohs, radioterapia, aplicación tópica de 5-fluoruracilo, fototerapia dinámica e incluso interferón- $\alpha$ y $\gamma$ vía sistémica e intralesionalmente.

En el 2001, Mackenzzie-Word ${ }^{9}$ utiliza un tratamiento tópico a base de una imidazoquinolona (imiquimod) en 16 pacientes diagnosticados de enfermedad de Bowen extragenital, con una respuesta al tratamiento de un $93 \%$. 


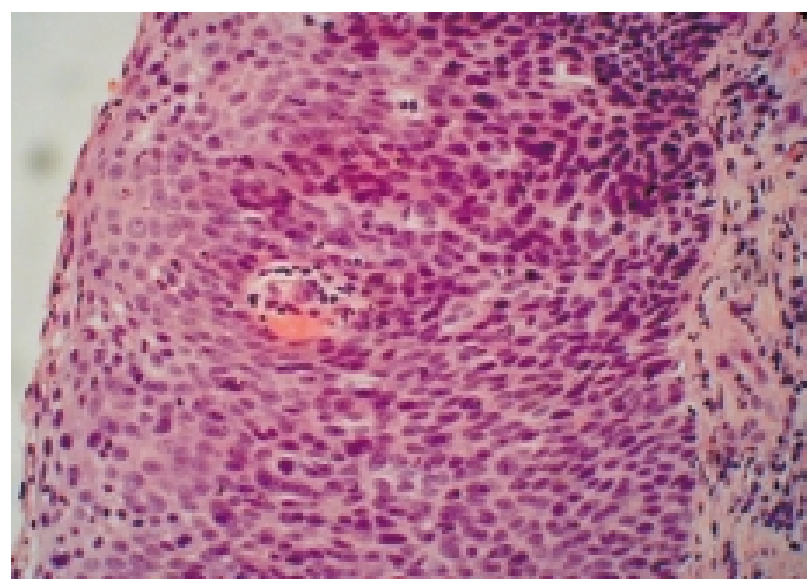

FIGURA 2. Neoplasia intraepitelial de pene. H. E. 400x.

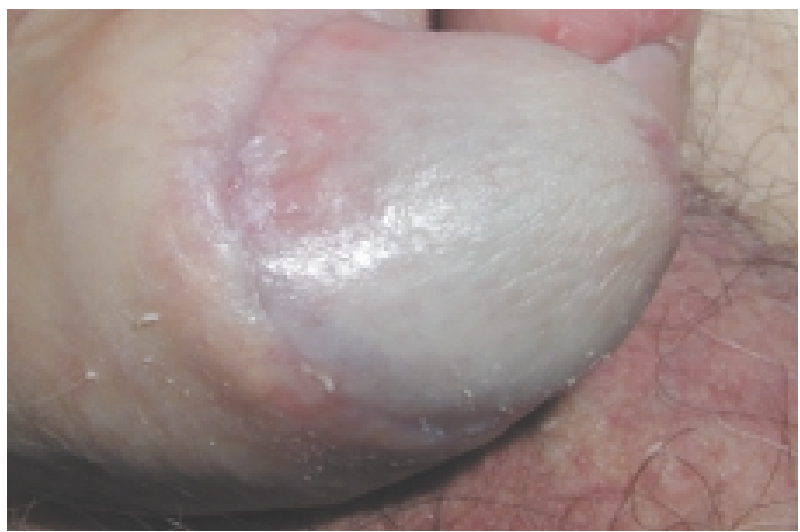

FIGURA 3. Aspecto estético tras tratamiento tópico de la lesión.

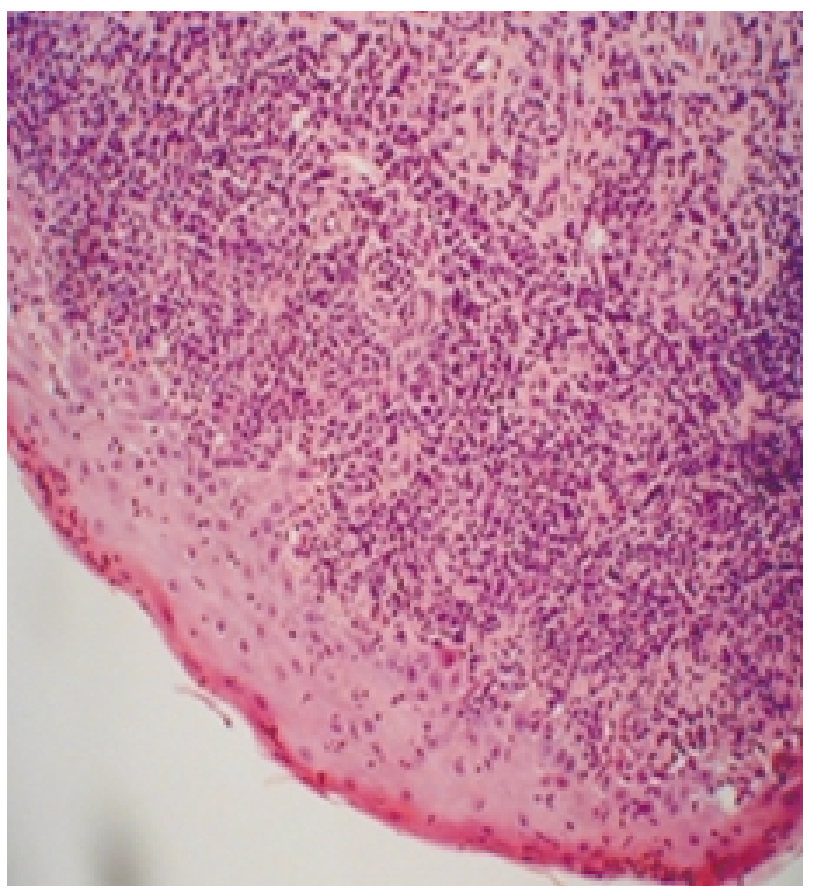

El imiquimod constituye un modulador de la respuesta inmune ${ }^{10}$ que actúa activando macrófagos y otras células mediante su unión a receptores de superficie, induciendo la secreción de citoquinas pro-inflamatorias del tipo interferón $\alpha$, factor de necrosis tumoral $\alpha$ e interleuquina $2 \mathrm{y}$ otras del tipo interleuquina $1 \alpha, 6,8,12$, interferón $\gamma$.

Asimismo esta imidazoqinolona parece presentar una actividad antiviral indirecta relacionada con un aumento de la síntesis de $2^{-}-5^{-}$-oligosintetasa ${ }^{11}$, lo que la hace particularmente efectiva en el tratamiento de las infecciones virales genitales.

$\mathrm{Su}$ aplicación se realiza tópicamente, sin vendaje oclusivo, 3 días a la semana durante 5 semanas. Los efectos adversos más frecuentes suelen ser reacciones cutáneas locales del tipo prurito, eritema, descamación, erosiones, ulceraciones, sinequias prepuciales, siendo en general bien toleradas ${ }^{12-19}$.

Hasta la fecha no son muchas las referencias encontradas sobre el uso de imiquimod tópico para el carcinoma in situ de pene, y ninguna de ellas en la literatura urológica; el primer caso fue aportado por Cook-Bolden ${ }^{12}$ con un seguimiento de 3 meses sin recidiva al igual que Danielsen ${ }^{13}$, Thai ${ }^{14}$ presenta un nuevo paciente con 6 meses de seguimiento, Orengo ${ }^{15}$ aporta un caso más con

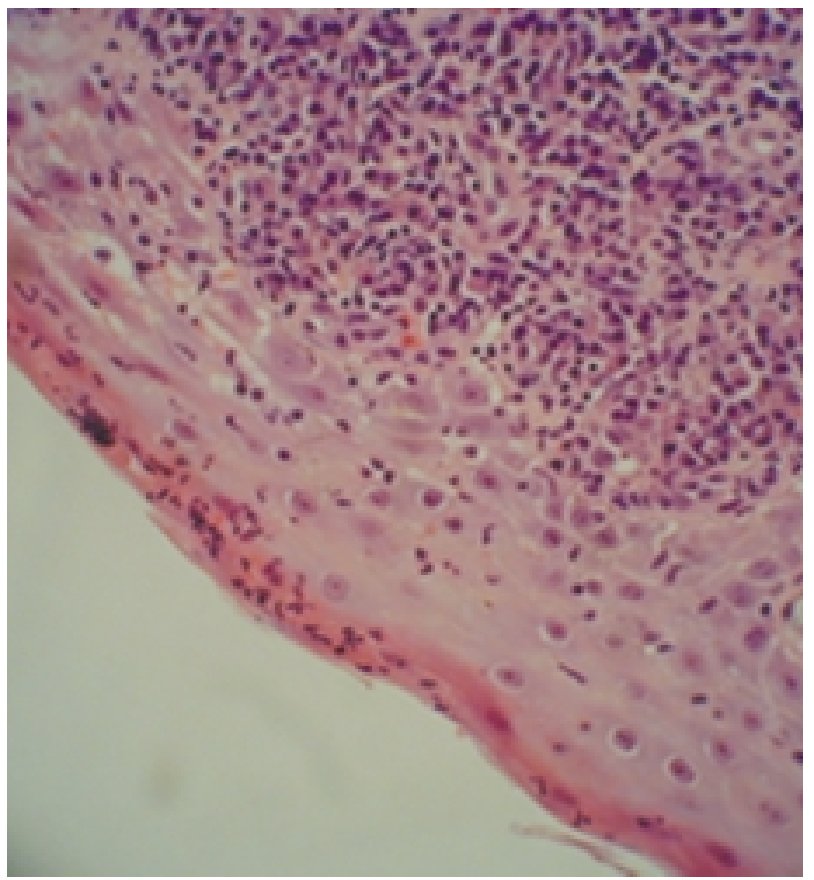

FIGURA 4. Ausencia de células atipicas, con reacción inflamatoria crónica. H. E. 200 y 400x. 
14 meses de evolución, Schroeder ${ }^{16}$ describe un paciente con buena respuesta al tratamiento sin evidencia clínica de recidiva a los 18 meses, Arlette $^{17}$ presenta la mayor serie de 5 pacientes con un seguimiento medio entre 3 y 12 meses, Micali ${ }^{18}$ aporta 2 casos más sin recidiva a los 4 y 6 meses y por último, Kaspari hace referencia al uso de imiquimod para un caso de Eritroplasia de Queyrat.

\section{CONCLUSIONES}

Pensamos que la aplicación tópica de imiquimod 5\% puede representar una alternativa más de tratamiento para las neoplasias intraepiteliales del pene por su fácil aplicación, escasos efectos secundarios y excelentes resultados estéticos y funcionales, precisando un seguimiento el paciente al igual que cualquier otra modalidad de tratamiento y la confirmación mediante biopsia profunda de la lesión y de sus bordes de la ausencia de carcinoma.

En caso de que el paciente no esté circuncidado creemos aconsejable su realización al tiempo que se practica la biopsia, por las sinequias que la aplicación de la crema puede producir, contribuyendo a la vez al tratamiento si la localización es prepucial y también a disminuir la posibilidad de recidivas.

\section{REFERENCIAS}

1. Gerber GS. Carcinoma in situ of the penis. J.Urol 1994;151: 829-833.

2. Kaye V, Zhang G, Dehner LP, Fraley EE. Carcinoma in situ of the penis. Is distinction between erythroplasia of Queyrat and Bowen`s disease relevant? Urology 1990;36:479-482.

3. Malek RS, Goellner JR, Smith TF, Espy MJ, Cupp MR. Human papillomavirus infection and intraepithelial, in situ and invasive carcinoma of penis. Urology 1993;42:159-170.

4. Cupp MR, Malek RS, Goellner JR, Smith TF, Espy MJ. The detection of human papillomavirus deoxyribonucleic acid in intraepithelial, in situ, verrucous and invasive carcinoma of the penis. J. Urol 1995;154:1024-1029.

5. Gómez I, Gómez E, Conde S, Maganto E, Navio S, Allona A. Infección por Papilloma virus en el hombre. Estado actual. Actas Urol Esp 2005;29:365-372.
6. Wieland U, Jurk S, Weissenborn S, Krieg T, Pfister H, Ritzkowsky A. Erytroplasia of Queyrat:coinfection with cutaneous carcinogenic human papillomavirus type 8 and genital papillomaviruses in a carcinoma in situ. J. Invest. Dermatol 2000;115:396-401.

7. Cox NH, Eedy DJ, Morton CA. Guidelines for the management of Bowen`s disease. Br J Dermatol 1999;141:633-641.

8. Buechener SA. Common skin disorders of the penis. BJU Int. 2002;90:498-506.

9. Mackenzie-Wood A, De Kossard S, Launey J, Wilkinson B, Owens ML. Imiquimod 5\% cream in the treatment of Bowen`s disease. J. Am. Acad. Dermatol 2001;44:462-470.

10. Stanley MA. Imiquimod and the imidazoquinolones:mechanism of action and therapeutic potential. Clin. Exp. Dermatol 2002;27:571 -577.

11. Diaz-Arrastia C, Arany I, Robazetti SC, Dinh TV, Gatalica $Z$, Tyring SK, Hanningan E. Clinical and molecular responses in high-grade intraepithelial neoplasia treated with topical imiquimod 5\%. Clin Cancer Res 2001;7:30313033.

12. Cook-Bolden F, Weinberg JM. Topical imiquimod 5\% cream in the treatment of Bowen's disease of the penis. $J$ Am Acad Dermatol 2002;46:146-147.

13. Danielsen AG, Sand C, Wweissman K. Treatment of Bowen's disease of the penis with imiquimod $5 \%$ cream. Clin Exp Dermatol 2003;28:7-9.

14. Thai Ke, Sinclair RD. Treatment of Bowen's disease of the penis with imiquimod. J Am Acad Dermatol 2002;46:470471.

15. Rengo I, Rosen T, Guill CK. Treatment of squamous cell carcinoma in situ of the penis with $5 \%$ imiquimod cream a case report. J Am Acad Dermatol 2002;47:S 225-228.

16. Schroeder TL, Sengelmann RD. Squamous cell carcinoma in situ of the penis successfully treated with imiquimod 5\% cream. J Am Acad Dermatol 2002;46:545-548.

17. Arlette JP. Treatment of Bowen's disease and erythroplasia of Queyrat. Br J Dermatol 2003;149 (suppl. 66):43-49.

18. Icali G, Nasca MR, Tedeschi A. Topical treatment of intraepithelial penile carcinoma with imiquimod. Clin Exp Dermatol 2003;28 (suppl.1):4-6.

19. Kaspari M, Gutzmer R, Kiehl P, Dumke P, Kaap A, Brodersen JP. Imiquimod 5\% cream in the treatment of human papillomavirus-16-positive erythroplasia of Queyrat. Dermatology 2002;205:67-69.

Dr. E. de Diego Rodríguez.

C/ Miguel de Unamuno, 6, 5ํㅡ.

39012 Santander (Cantabria)

e-mail:e.diego@telefonica.net

(Trabajo recibido el 10 mayo 2005) 